\title{
Answer to the Letter to the Editor of Hamid Namazi concerning manuscript "Platelet-rich plasma in mono-segmental posterior lumbar interbody fusion”. Eur Spine J, 2011 Jul 10 [Epub ahead of print], by J. Sys et al.
}

Jan Sys

Accepted: 18 August 2011 / Published online: 1 September 2011

(C) Springer-Verlag 2011

Indeed, Platelet-rich plasma (PRP) can stimulate osteoprotegerin production which can lead to a decreased chemotaxis of osteoclasts. PRP can induce the production of vascular endothelial growth factor (VEGF), leading to inhibition of osteoclast differentiation. Both mechanisms are well documented.

Besides VEGF, platelet-derived growth factor (PDGF), and transforming growth factor beta (TGF- $\beta$ ) are among the known growth factors to be important in bone healing. PRP can influence the concentration of these factors.

The concept of PRP application is to enhance the healing properties of bone by stimulating differentiation and proliferation of osteoblasts and/or by inhibiting osteoclast activity. Because multiple growth factors, cytokines, and platelet proteins are present at the bone formation site, a synergistic effect is assumed.

Basic science literature strongly supports these positive effects of PRP on bone healing. In contrast, evidence from prospective clinical studies is lacking. Because the eventual clinical benefit in spinal fusion is expected to be small, large-size studies are needed. In this underpowered study we only could notice a "tendency" of better scores in VAS and SF-36 (PCS-subscore) in the PRP group. The discrepancy between basic science evidence and clinical evidence remains. 